\title{
Dimensioning and Location Planning for Wireless Networks under Multi-level Cooperative Relaying
}

\author{
Bin $\operatorname{Lin}^{1,2}$ and Pin-Han $\mathrm{Ho}^{1}$ \\ 1 Department of Electrical and Computer Engineering, \\ University of Waterloo, Waterloo, ON N2L 3G1, Canada \\ 2 Department of Information Technology, \\ Dalian Maritime University, Dalian, China, 116026 \\ \{b2lin, pinhan\}@bbcr. uwaterloo.ca
}

\begin{abstract}
This paper studies the problem of network dimensioning and location planning in multi-hop wireless networks. A key technology of wireless multi-level cooperative relaying $(\mathrm{CR})$ is incorporated, which has been recognized as an effective design paradigm for achieving throughput enhancement. A mathematical formulation is presented to capture the nature of the problem, and characterize the behavior of multi-level CR. The tasks of dimensioning, relay placement, relay allocation, and relay sequence design have been considered in a unified optimization framework. The formulation is a nonlinear integer program. To avoid the intractable computation complexity in solution, an efficient two-phase algorithm is developed. We conduct a series of case studies to verify the proposed algorithm, in which the results demonstrate the efficiency of our proposed algorithm and the significant benefits in terms of deployment cost reduction under multi-level CR.
\end{abstract}

Keywords: Networking Dimensioning and Location Planning, Multilevel Cooperative Relaying, Optimization.

\section{Introduction}

Network planning is a never-ending process with the wireless network's evolution and growth. A new round of work is forced when it is needed to meet new requirements of boosting system throughput and/or changing of network environments 1]. Typically, new shopping centers and communities in a cell or at a cell's edge may lead to new hotspots or demand for new (micro-) cell sites, and newly constructed buildings may change the multipath environments, possibly resulting in "black holes" in the coverage area. To address these newly arisen problems in existing wireless networks, placing relay stations (RSs) between a base station (BS) and subscriber stations (SSs) provides a viable approach, instead of the conventional setup of a new (micro-) cell by deploying BS. The quality of wireless channels can be improved by multi-hop relaying. And RSs can be placed close to black holes, as they can establish extra transmission

L. Fratta et al. (Eds.): NETWORKING 2009, LNCS 5550, pp. 207-219, 2009.

(C) IFIP International Federation for Information Processing 2009 
links to circumvent obstacles. Moreover, compared with BSs, RSs can be deployed more easily, faster, and cheaper, which are rather appealing to network operator.

In this paper, we study the problem of Dimensioning and Location Planning (DLP) of RSs in multi-hop wireless networks. A key technology of multi-level cooperative relaying $(C R)$ is incorporated since cooperative communication has been recognized as an effective paradigm for achieving throughput enhancement. Thus, incorporating promising technical breakthrough will lead to new problem, new model and new solution so as to achieve a cost-effective design.

The multi-level $C R$ in this paper refers to a multi-hop transmission that both the source and internal relays cooperatively transmit to a destination. It is different from the traditional multi-hop non-cooperative transmission (NCT), in which the received data are simply forwarded at each internal nodes to the nexthop node. In other words, with multi-level $C R$, the destination receives multiple copies of data packets (possibly in different codes) from the source and all the upstream relays; while, with traditional multi-hop NCT, the destination only receives one copy of data packet from its previous-hop node. Therefore, multilevel CR transmission can achieve even higher throughput. Moreover, multiple simultaneous active links with multi-level $C R$ can provide better robustness and fault tolerance than a single link.

However, the relay locations have a critical effect on the destination's achievable rate 2, 3, even in the simplest relay model with only 3 nodes (source-relaydestination) [4]. In wireless networks with multi-level $\mathrm{CR}$, deciding the location of each RS and estimating the amount of RSs needed are much more challenging. In literatures, numerous research efforts have been undertaken to address the placement problems. So et. al studied minimum cost configuration of relay and channel infrastructure in heterogeneous wireless mesh networks (WMNs) in [5], where Bender's decomposition-based heuristic was proposed to solve it efficiently. The placement of tetherless relay points (TRPs) to improve the throughput of a WLAN was investigated in [6], in which a Lagrangian relaxation iterative algorithm was developed. In [7] and [8], for planning WMNs, Amaldi et. al presented a novel optimization model and a relaxation-based heuristic. The issues of traffic routing, interference, rate adaptation, and channel assignment were also considered.

In this paper, we develop an optimization framework for the DLP problem. A mathematical model is provided to capture the nature of DLP problem, and characterize the behaviors and constraints under multi-level CR. We have jointly considered RS placement, relay allocation, and relay sequence design in a unified framework. The formulation is an integer nonlinear program, which is nonetheless subject to intractable computation complexity in solution. Thus, a two-phase algorithm is developed for solving the problem effectively and efficiently. Simulation and case studies are conducted to verify the optimization framework and demonstrate the economic and performance benefits of the multi-level CR against traditional multi-hop NCT. 


\section{Modeling of Multi-level Cooperative Relaying}

In the fundamental multi-level CR model, a source (node 0) communicates with a destination (node $G$ ) through multiple relays (node $1,2, \ldots, G-1$ ). The achievable rate for a fixed destination can be expressed as 9 ]

$$
R=\min _{1 \leq j \leq G}\left[C\left(\frac{1}{N_{0}} \sum_{k=1}^{j}\left(\sum_{i=0}^{k-1} \sqrt{P_{i k}} d_{i j}^{-\alpha}\right)^{2}\right)\right]
$$

where $\alpha$ is the path loss exponent, $P_{i k}$ is the transmit power of node $i$ to node $k, N_{0}$ is the power of the background noise, $C($.$) is the Shannon function such$ that $C(x)=\frac{1}{2} \log (1+x)$ for $x \geq 0$, with $d_{i j}$ as the distance between node $i$ and $j$. In this paper, we assume that all the nodes use the same power density for transmission, i.e., $P_{i k}=P_{0}$ for $\forall i, k$. The more complex issue of power control will be deferred for future research.

\section{Network Model and Problem Formulation}

\subsection{Network Model}

A three-tier network architecture is considered, which consists of three network entities: a BS, RSs, and fixed SSs. The BS serves as a central controller in the cell. The RSs are responsible for relaying data between the BS and the associated SSs under multi-level CR [9]. The RSs have no direct connections to the core network. Within a cell, there exists a certain number of new SSs, which could be a residential or business subscriber. Each SS submits a certain data rate requirement. These information must be known prior to the network planning, and they can be acquired by statistical data analysis of traffic measurement as well as anticipation of future growth. Since an RS cannot be placed anywhere due to some geographic limitations, certain physical locations in the cell are taken as candidate positions (CPs) for placing RSs. The COST231-Hata model is adopted as the channel model which is applicable to the transmissions inside an urban environment [10. Small scale fading is not explicitly included in this study since a long-term planning and design is targeted. Additionally, in consideration of transmission delay, the maximum number of allowable intermediate RSs between the BS and an SS in a cell is assumed to be limited.

\subsection{Problem Formulation}

We consider a practical deployment scenario in a broadband wireless access network. The goals of the DLP problem are

(i) to obtain the minimal number of RSs to be deployed;

(ii) to obtain the optimal locations of the RSs,

(iii) to allocate serving $\mathrm{RS}(\mathrm{s})$ to each $\mathrm{SS}$,

(iv) to determine the relay sequence of the serving $\mathrm{RS}(\mathrm{s})$ for each $\mathrm{SS}$, 
Table 1. Definitions of important symbols

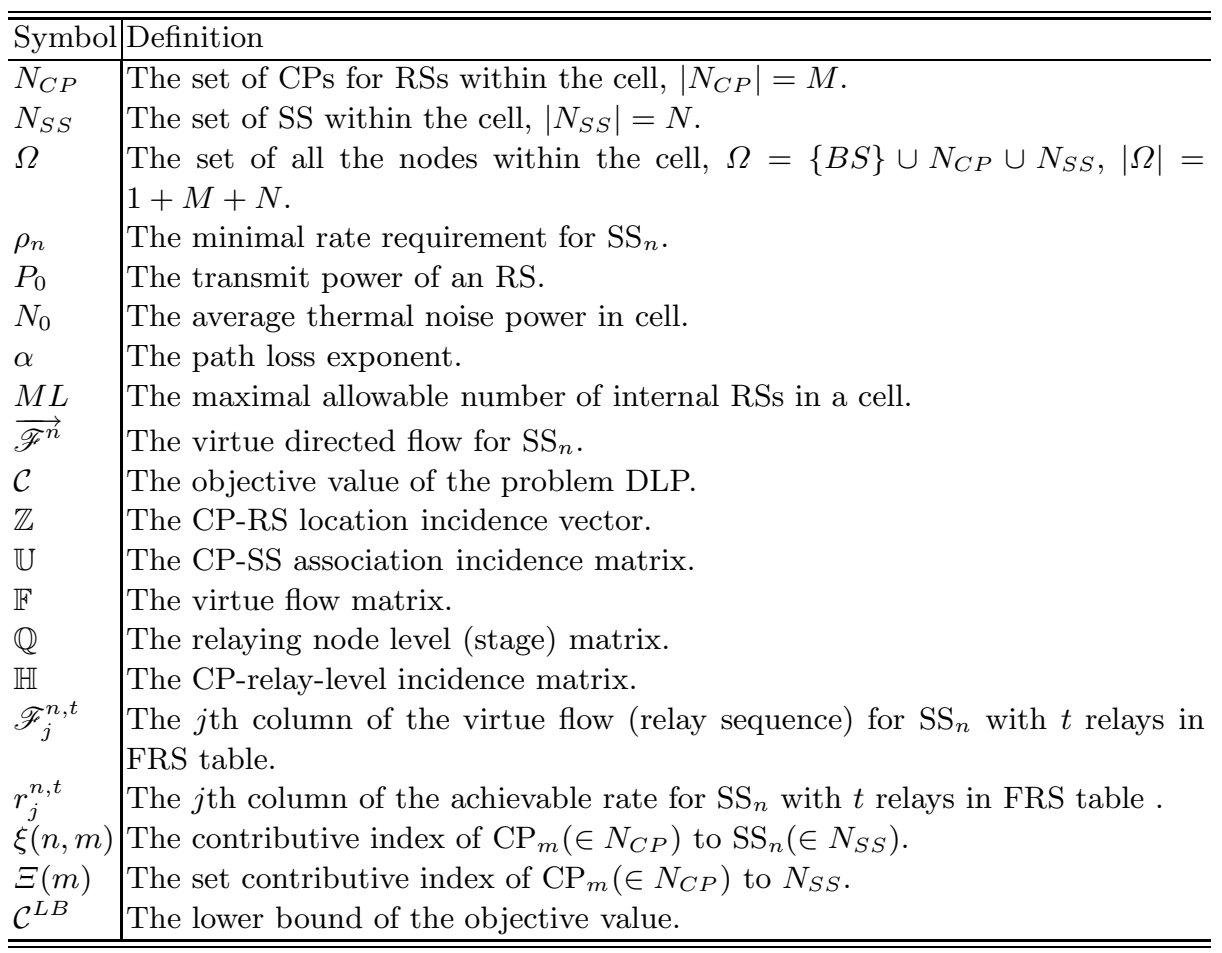

such that each SS's minimal rate requirement is satisfied. The notations used in the problem are listed in Table 1

Let $\vec{G}=(\Omega, \vec{E})$ represent the directed graph, which consists of a node set $\Omega=\left\{B S \bigcup N_{C P} \bigcup N_{S S}\right\}$ and a directed edge set $\vec{E} . N_{C P}$ and $N_{S S}$ are the set of CPs and SSs, respectively. To formulate the problem, we start by defining the decision variables for $\mathrm{RS}$ location $(\mathbb{Z})$ and allocation $(\mathbb{U})$, respectively. $\mathbb{Z}=$ $\left(z_{m}\right)_{1 \times M}$ is a CP-RS incidence vector (location variable) where $z_{m}=1$ if $C P_{m}$ is selected for placing an RS; otherwise, $z_{m}=0$. $\mathbb{U}=\left(u_{m n}\right)_{M \times N}$ is a CP-SS incidence matrix ( $C P$ allocation variable) where $u_{m n}=1$ if $\mathrm{CP}_{m}$ is allocated to serving $S S_{n}$; otherwise, $u_{m n}=0$.

Since the DLP problem targets at minimizing the total number of RSs, denoted by $\mathcal{C}$, the objective function can be expressed as,

$$
\operatorname{minimize} \quad \mathcal{C}=\sum_{m \in N_{C P}} z_{m}
$$

The RS location and allocation variables (i.e., $\mathbb{Z}$ and $\mathbb{U}$ ) are subject to the following constraints.

$$
u_{m n} \leq z_{m}, \quad \forall m \in N_{C P}, n \in N_{S S}
$$




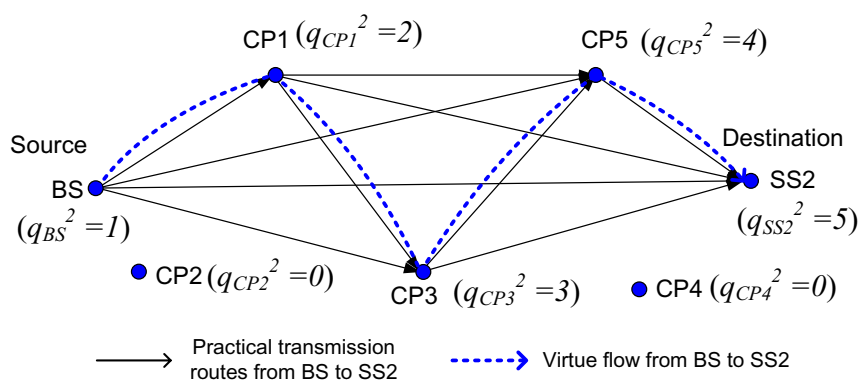

Fig. 1. An illustration of practical relay routes and virtue flow $\left(\mathrm{BS} \rightarrow \mathrm{SS}_{2}\right)$

$$
\sum_{m \in N_{C P}} u_{m n} \leq M L, \quad \forall m \in N_{C P}, n \in N_{S S} \in N_{S S}
$$

Constraint (3) ensures that if $\mathrm{CP}_{m}$ is associated with $\mathrm{SS}_{n}$, an RS must be placed at $\mathrm{CP}_{m}$. Constraint (4) makes a limit for the maximal number of internal relays (i.e., associated CPs) for an SS.

The complexity of the formulation arises at representing the achievable rate for each SS in practical deployment scenario. It is because each CP is still undetermined whether to place an RS and whether to be allocated to an SS. Moreover, even the allocation of RSs to an SS has been determined, their exact sequence in a relay route are still undecided, since there are totally $n$ ! permutations of possible sequences for $n$ RSs. By observing (11), we find that the destination's achievable rate heavily depends on the locations and relay sequence of the allocated RSs. To represent the relay sequence for each SS, we adopt the concept of virtue flow, which is defined as the route with the maximal number of hops among all the practical transmission routes from $\mathrm{BS}$ to $\mathrm{SS}_{n}$, denoted by $\overrightarrow{\mathscr{F}^{n}}$. As an example shown in Figure 1, suppose $\mathrm{CP}_{1}, \mathrm{CP}_{3}$ and $\mathrm{CP}_{5}$ are allocated to provision transmissions to $\mathrm{SS}_{2}$, while $\mathrm{CP}_{2}$ and $\mathrm{CP}_{4}$ are not. The virtue flow (relay sequence) from BS to $\mathrm{SS}_{2}\left(\overrightarrow{\mathscr{F}^{2}}\right)$ is illustrated with a blue dotted line. Namely, $\overrightarrow{\mathscr{F}^{2}}:\left\{\mathrm{BS}, \mathrm{CP}_{1}, \mathrm{CP}_{3}, \mathrm{CP}_{5}, \mathrm{SS}_{2}\right\}$, for simplicity, $\overrightarrow{\mathscr{F}^{2}}:\left\{\mathrm{CP}_{1}, \mathrm{CP}_{3}, \mathrm{CP}_{5}\right\}$.

Then, we define a virtue flow matrix $\mathbb{F}=\left(f_{i j}^{n}\right)_{(M+1) \times(M+1) \times N}$, where $f_{i j}^{n}=1$ if both node $i$ and node $j$ are in $\overrightarrow{\mathscr{F}^{n}}$, and node $i$ is on the upstream of node $j$ (for $i \in B S \bigcup N_{C P}, j \in N_{C P} \bigcup S S_{n}, n \in N_{S S}$ ); otherwise, $f_{i j}^{n}=0$. For each SS, the virtue flow matrix is subjected to :

$$
\begin{aligned}
& f_{i j}^{n}+f_{j i}^{n} \leq 1, \quad \forall i, j \in N_{C P}, n \in N_{S S} \\
& \sum_{m \in N_{C P}} f_{B S, m}^{n}=1, \quad \forall n \in N_{S S} \\
& \sum_{i \in \Omega} f_{i, S S_{n}}^{n}=1, \quad \forall n \in N_{S S}
\end{aligned}
$$




$$
\begin{aligned}
& \sum_{i \in N_{C P} \cup B S} f_{m i}^{n}=u_{m n}, \quad \forall m \in N_{C P}, n \in N_{S S} \\
& \sum_{i \in N_{C P} \cup B S} f_{i m}^{n}=u_{m n}, \quad \forall m \in N_{C P}, n \in N_{S S} \\
& \sum_{j_{1} \in N_{C P}} f_{j_{1} m}^{n}+\sum_{j_{2} \in N_{C P}} f_{m j_{2}}^{n}=2 u_{m n}, \quad \forall m \in N_{C P}, n \in N_{S S}
\end{aligned}
$$

Constraint (5) stipulates the virtue flow is directed where $f_{i j}^{n}=1$ if node $i$ is on the upstream of $j$ in $\overrightarrow{\mathscr{F}}^{n}$. Constraints (6) and (7) ensure that the BS and each terminal node $\left(\mathrm{SS}_{n}\right)$ in $\overrightarrow{\mathscr{F}^{n}}$ has only one downstream node and one upstream node, respectively. Constraints (8) and (9) ensure that each CP in $\overrightarrow{\mathscr{F}} n$ also has exactly one upstream node as well as one downstream node, if the CP is associated with $\mathrm{SS}_{n}$ (i.e., the CP is in $\overrightarrow{\mathscr{F}^{n}}$ ). Constraint (10) formulates the flow conservation property; i.e., the number of upstream and downstream nodes of a CP equals two if the CP is in $\overrightarrow{\mathscr{F}^{n}}$.

To represent the position of a node in a relay sequence, we now define the relay level of a node as below.

Definition 1. Relay level. The relay level of a node $A$ for a destination $D$ (denoted by $q_{A}^{D}$ ) is defined as $q_{A}^{D}=\sum_{A \in \Omega} \operatorname{deg}^{-}(A)+1$,where $\sum_{A \in \Omega} \operatorname{deg}^{-}(A)$ represents the indegree of node $A$ in graph $G$. Note that $q_{A}^{D}=0$ indicates that node $A$ is not associated with destination $D$.

In Figure 1, the relay levels of $\mathrm{BS}, \mathrm{CP}_{1}-\mathrm{CP}_{5}$ are 1, 2, 0, 3, 0 and 4, respectively.

We define a node relay level matrix $\mathbb{Q}=\left(q_{i}^{n}\right)_{|\Omega| \times N}$, where $q_{i}^{n}$ is the relay level of node $i \in \Omega$. According to the constructed directed virtue flow, the relay level of the BS should always be 1 . The relay level of node $j$ increases by one, if both $i$ and $j$ are in $\overrightarrow{\mathscr{F}}^{n}$ and the upstream node of $j$ is $i$; otherwise, the relay level is set to 0 . The following constraints (11)-(13) stipulate the above definition of relay level.

$$
\begin{aligned}
& q_{B S}^{n}=1, \quad n \in N_{S S} \\
& q_{j}^{n}-q_{i}^{n} \geq f_{i j}^{n}-\beta\left(1-f_{i j}^{n}\right), \forall i \in N_{C P} \cup B S, j \in N_{C P} \cup S S_{n}, n \in N_{S S} \\
& q_{i}^{n} \geq 0, i \in \Omega, n \in N_{S S}
\end{aligned}
$$

where $\beta=|\Omega|$. To establish a connection between the relay level of a CP and Eq. (1), a new set of variable $\mathbb{H}$ is needed. $\mathbb{H}=\left(H_{h i}^{n}\right)_{M L \times M \times N}$ is a CP-relay-level incidence matrix such that $H_{h i}^{n}=1$ if node $i \in N_{C P}$ is in $\overrightarrow{\mathscr{F}}^{n}$ and $i$ 's relay level is $h \in\{2, \ldots, M L+1\}$; otherwise, $H_{h i}^{n}=0$. The following constraints (14) and (15) stipulate the definition of $\mathbb{H}$. Constraint (16) ensures that there is at most one node at each level in $\overrightarrow{\mathscr{F}}^{n}$.

$$
H_{h i}^{n} \geq \gamma+\gamma\left(q_{i}^{n}-h\right), \forall i \in N_{C P}, n \in N_{S S}, h \in\{2, \ldots, M L+1\}
$$




$$
\begin{array}{ll}
1-H_{h i}^{n} \geq \gamma+\gamma q_{i}^{n}, & \forall i \in N_{C P}, n \in N_{S S}, h \in\{2, \ldots, M L+1\} \\
\sum_{i \in N_{C P}} H_{h i}^{n} \leq 1, & \forall n \in N_{S S}, h \in\{2, \ldots, M L+1\}
\end{array}
$$

where $\gamma=\frac{1}{|\Omega|^{2}}$. To ensure the throughput of each SS is larger than the minimal rate requirement, the constraints can be expressed as follows.

$$
\begin{aligned}
& \log \left[1+\left(\frac{1}{N_{0}} \sum_{k=2}^{h_{2}}\left(\sum_{h_{1}=1}^{k} \sqrt{P_{0}}\left(D_{h_{1} h_{2}}^{n}\right)^{-\alpha}\right)^{2}\right)\right] \geq \rho_{n}, h_{2}=2 \ldots M L+1, \forall n \in N_{S S} \\
& D_{h_{1} h_{2}}^{n}=\sqrt{\left(\sum_{l \in \Omega} H_{h_{1} l}^{n} x_{l}-\sum_{l \in \Omega} H_{h_{2} l}^{n} x_{l}\right)^{2}+\left(\sum_{l \in \Omega} H_{h_{1} l}^{n} y_{l}-\sum_{l \in \Omega} H_{h_{2} l}^{n} y_{l}\right)^{2}} \\
& \quad \forall 2 \leq h_{1}<h_{2} \leq M L+1, \forall n \in N_{S S} \\
& D_{1, h_{1}}^{n} \leq \frac{D_{1, h_{2}}^{n}}{\varepsilon+\sum_{l \in \Omega} H_{h_{2} l}}, \forall 2 \leq h_{1}<h_{2} \leq M L+1, n \in N_{S S}
\end{aligned}
$$

where $\varepsilon$ is a very small number. Constraint (19) ensures that the relay sequence of the internal relays (the selected CPs) is the one with maximal achievable rate among all the permutations of the nodes.

In summary, the objective function (2) and the constraints (3)-(19) form the mathematical formulation for problem DLP. Due to the nonlinearity of constraints (17), (18) and (19), problem DLP is a nonlinear integer program, which cannot be solved by any systematic method [11. In the next section, an effective algorithm will be presented by exploiting the nature of the problem.

\section{An Algorithm to Solve Problem DLP}

\subsection{Algorithm Description}

We propose a two-phase algorithm to solve problem DLP, which is shown in Algorithm 1. The core idea is as follows. First, we reduce the search space such that only feasible candidate relay sequences are left. Then, in Phase II, the best relay sequence for each SS can quickly be found by iteratively removing the least contributive CP.

\section{- Phase I: Feasible-Relay-Sequence (FRS) Table Setup Phase}

The search space is defined in the FRS table, which is the basis for deciding the RSs' final locations in the next phase. As an example, assume $M L=3$, the FRS table is shown in Figure 2, Each SS has its own sub-table. The sub-table consists of two rows: a row of achievable rates, which are sorted in non-increasing order, and a row of relay sequences, which one-to-one map the rates in the same column in the first row. $r_{j}^{n, t}, \mathscr{F}_{j}^{n, t}$ represent the achievable rate of $\mathrm{SS}_{n}$ via $t$ relays, and the corresponding relay sequence (i.e., virtue flow) for $\mathrm{SS}_{n} \in N_{S S}$, respectively. $j$ is the column index. 


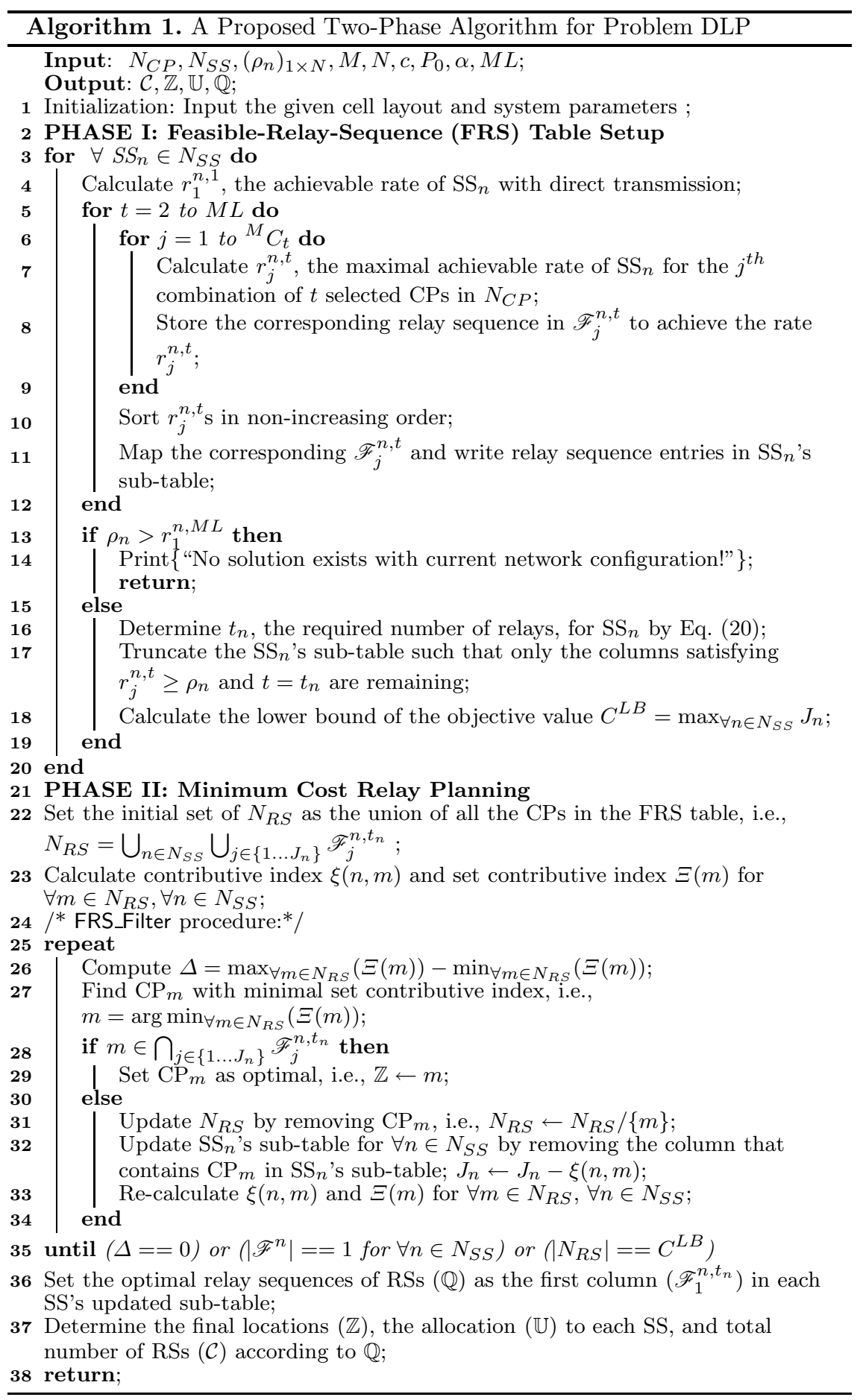




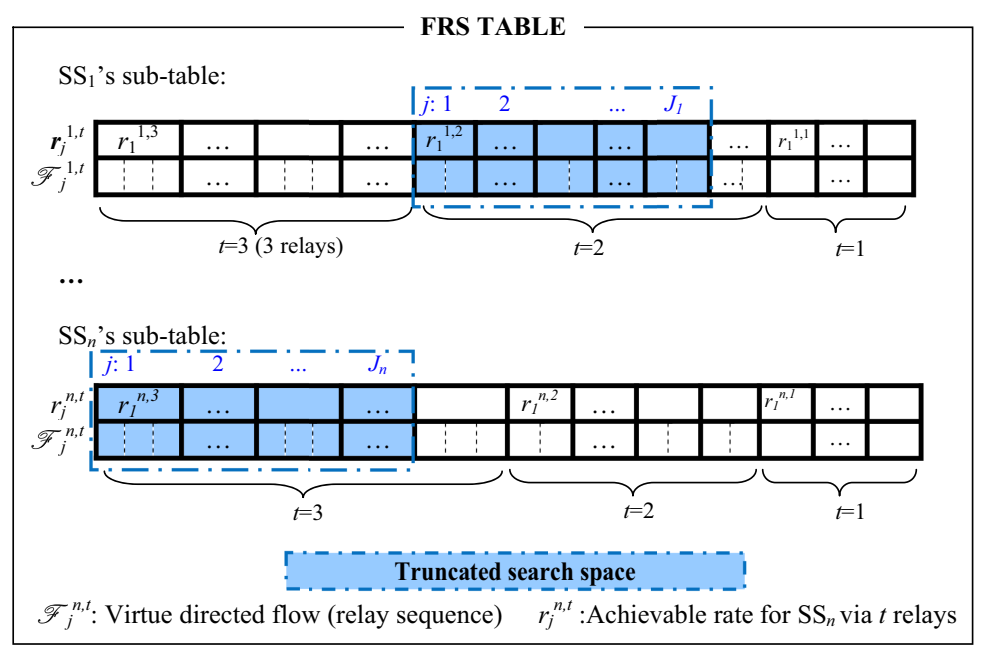

Fig. 2. An illustration of FRS table $(M L=3)$

Phase I focuses on setting up such a FRS table. To construct it, for each SS, $r_{j}^{n, t}$ is calculated with Eq. (11) by enumerating all the possible combinations of $t$ relays $(\mathrm{CPs})$ in $N_{C P}$, where $t=\{1 \ldots M L\}$. Note that there are $t$ ! number of permutations for a specific set of $t$ relays, which lead to $t$ ! achievable rates. Thus, the maximal one is set as $r_{j}^{n, t}$ and the corresponding relay sequence is stored in $\mathscr{F}_{j}^{n, t}$. Then, in Line 10, we sort all the $r_{j}^{n, t} \mathrm{~s}$ in non-increasing order, and map the corresponding $\mathscr{F}_{j}^{n, t}$. The entries of $r_{j}^{n, t}$ s and $\mathscr{F}_{j}^{n, t}$ s are then written in the FRS table. In Line 13, we check if there exists a feasible solution with current network configuration (the set of CPs). More specifically, we compare $\rho_{n}$ and $r_{1}^{n, M L}$, which is the maximum possible rate for $\mathrm{SS}_{n}$. If $\rho_{n}>r_{1}^{n, M L}$, it indicates more better CPs are needed in $N_{C P}$. If $\rho_{n} \leq r_{1}^{n, M L}$ for $\forall n$, there must exist a feasible solution. By exploiting the monotonicity of achievable rate row in $\mathrm{SS}_{n}$ 's sub-table, we can use the following criteria to determine the required number of relays (denote as $t_{n}$ ) for $\mathrm{SS}_{n}$ to achieve its minimum rate requirement.

$$
t_{n}=i+1, \text { if } r_{1}^{n, i+1} \geq \rho_{n}>r_{1}^{n, i}, \forall n \in N_{S S}(i=0, \ldots, M L-1)
$$

Afterwards, we truncate the search space in each SS's sub-table such that only the columns satisfying $r_{j}^{n, t} \geq \rho_{n}$ and $t=t_{n}$ are remaining. $J_{n}$ is the total number of columns in $\mathrm{SS}_{n}$ 's sub-table after truncation.

\section{- Phase II: Minimum Cost Relay Planning Phase}

In this phase, we propose a FRS_Filter method to find the minimum cost placement, which is a procedure of iteratively removing the least contributive CP to $N_{S S}$.

Definition 2. Contributive Index. The contributive index of $\mathrm{CP}_{m}\left(\in N_{C P}\right)$ to $\mathrm{SS}_{n}\left(\in N_{S S}\right)$ (denoted as $\xi(n, m)$ ) is defined as the total number of $\mathrm{CP}_{m}$ in the relay sequence row of $\mathrm{SS}_{n}$ 's sub-table . 
Definition 3. Set Contributive Index. The set contributive index of $\mathrm{CP}_{m}(\in$ $N_{C P}$ ) to the set $N_{S S}$ (denoted as $\Xi(m)$ ) is defined as the total number of $\mathrm{CP}_{m}$ in all the relay sequence rows in the FRS table, i.e., $\Xi(m)=\sum_{n \in N_{S S}} \xi(n, m)$.

Remark. $\Xi(m)$ represents the contributive index of a specific $\mathrm{CP}_{m}$ to the whole set of $N_{S S}$. The smaller the value of $\Xi(m)$, the less chance that $\mathrm{CP}_{m}$ is selected to the final optimal set of RSs. Based on this concept, in Phase II, the minimal cost placement will be realized through the process of FRS_Filter.

In Line 22, we first get the initial set of RSs $\left(N_{R S}\right)$, which is the union of all CPs in the FRS table. Then, the $\xi(n, m) \mathrm{s}$ and $\Xi(m) \mathrm{s}$ for $\forall m \in N_{R S}, \forall n \in N_{S S}$ are calculated according to DEFINITION 2 and 3. In Lines 24-35, the procedure of FRS_Filter is executed. Suppose $\mathrm{CP}_{m}\left(\in N_{R S}\right)$ is the one with minimal set contributive index. We then check if it appears in each column of relay sequence in the $\mathrm{SS}_{n}$ 's sub-table. If yes, it indicates that $\mathrm{CP}_{m}$ must be selected to place an $\mathrm{RS}$ and it can not be removed, i.e., $\mathrm{CP}_{m}$ is an optimal location for RS; otherwise, we can update $N_{R S}$ by removing $\mathrm{CP}_{m}$. Then all the SSs' sub-tables should also be updated by removing the whole column that contains $\mathrm{CP}_{m}$. Afterwards, all the $\xi(n, m)$ s and $\Xi(m)$ s are re-calculated so as to conduct next loop of FRS_Filter operations. The loop terminates when any of the following is true: (1) $\Delta$ equals zero, where $\Delta=\max _{\forall m \in N_{R S}}(\Xi(m))-\min _{\forall m \in N_{R S}}(\Xi(m))$. It indicates that all the CPs left in the FRS table have an equal chance to be finally selected, namely, none is worse than the others. Thus, the current $N_{R S}$ is the optimal, and $\mathbb{Z}$ can be determined accordingly. (2) $\left|\mathscr{F}^{n}\right|$ equals one for $\forall n \in N_{S S}$. It indicates that there is only one column remaining in each SS's sub-table, in which the left relay sequence is definitely optimal. (3) $\left|N_{R S}\right|$ equals $C^{L B}$. It indicates that the lower bound of the objective value has been achieved, so the remaining relay sequences are definitely optimal.

\section{$5 \quad$ Numerical Results}

We consider a practical network scenario where an IEEE 802.16 WirelessMAN air interface [12 is assumed. $M L$ is set to 2 . Each node transmits with a unit power density. The path loss exponent $\alpha$ is set to 3 . To simulate the presence of buildings, trees and other obstructions in practical network environments, the shadowing effects typically result in 5-10 dB losses are also taken into account in the simulations. Without loss of generality, the BS is located at the origin point $(0,0)$. We conduct a series of case studies. Figure 3 shows one of the network layouts, in which the normalized coordinate of each SS and CP is illustrated and the amount of minimal rate requirement for each SS is proportional to its radius.

To verify the optimality of our proposed two-phase algorithm, we compare the results with the optimal one (obtained by an exhaustive search). We find that the final objective value of our proposed algorithm is the same with the optimum for all the cases we studied. Figure 3 also shows the resulting configurations of the RS placement and the optimal relay sequences (virtue flows), which are represented with the dotted line from BS to each SS.

The proposed algorithm demonstrates excellent computation efficiency as shown in Figure 4. The computation time of our proposed algorithm increases 


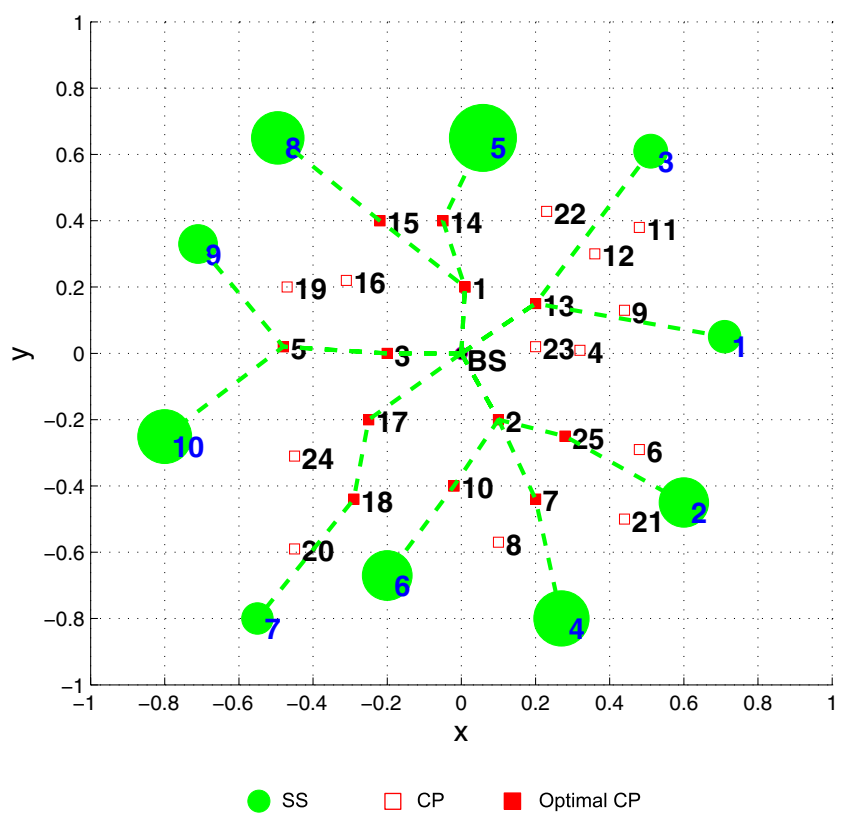

Fig. 3. The optimal results obtained by proposed 2-phase algorithm

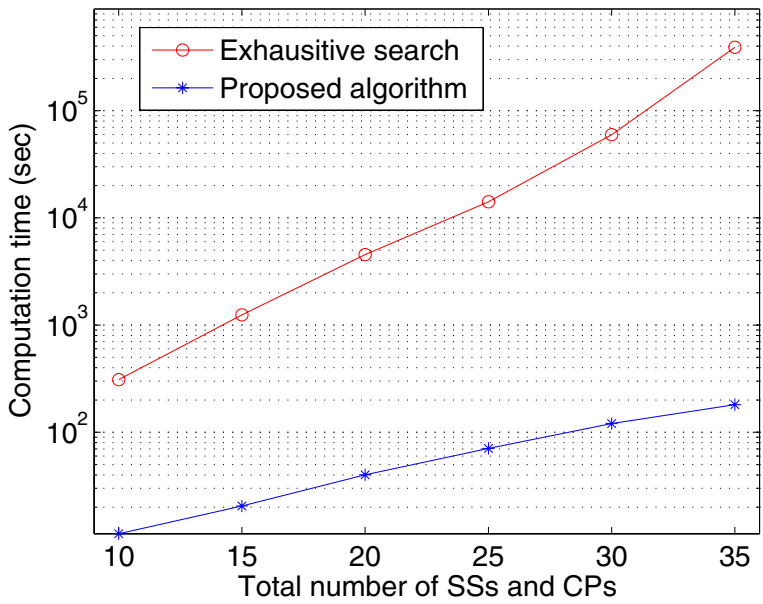

Fig. 4. The comparison of computation time of proposed algorithm against exhaustive search

linearly with the problem size (i.e., the total number of SSs and CPs), while the computation time shows an exponential growth with the exhaustive search. Therefore, our proposed algorithm shows a good scalability in practical largescale wireless network design. 
Table 2. Objective Value Comparison

\begin{tabular}{c|l|c|c|c|c|c|c|c}
\hline \hline & Number of SSs & 4 & 5 & 6 & 7 & 8 & 9 & 10 \\
\hline \hline \multirow{3}{*}{\begin{tabular}{c} 
Objective $\begin{array}{c}\text { o } \\
\text { value }\end{array}$ \\
\cline { 2 - 9 }
\end{tabular}} & multi-level CR & 5 & 7 & 8 & 9 & 9 & 10 & 12 \\
\cline { 2 - 9 } & Sulti-hop NCT & 8 & 12 & 15 & 17 & 19 & 22 & 24 \\
\hline \hline
\end{tabular}

Table 2 compares the objective value (i.e., the number of RSs) with multilevel CR against traditional multi-hop NCT. It is observed that the number of RSs to be deployed can be substantially reduced $(37.5 \% \sim 54.6 \%)$ when multi-level CR is employed. Therefore, by incorporating advanced cooperative transmission technology, a salient economic benefit in terms of RS deployment cost reduction can be achieved. On the other hand, given the same RS placement, a significant improvement on end-user and system throughput can be achieved with cooperative transmission, which just meets the ever-increasing demands of future residential and business premises.

\section{Conclusions}

In this paper, we have conducted a comprehensive study on the issue of dimensioning and location planning in wireless networks under multi-level CR. The tasks of RS placement, relay allocation, and relay sequence design have been jointly considered and formulated in a unified optimization framework. To avoid intractable computation complexity in solving the nonlinear formulation, a two-phase algorithm has been developed to solve it efficiently and effectively. Simulation and case studies have been conducted, in which the results have demonstrated the RS deployment cost can be reduce by $37.5 \% \sim 54.6 \%$ with multi-level CR compared with that with traditional multi-hop NCT. The proposed framework should provide a guideline for the operators in the effort of RS placement and evaluation of network expenditure in practice.

\section{References}

1. Korhonen, J.: Introduction to 3G Mobile Communications, 2nd edn., p. 251. Artech House (2002)

2. Lin, B., Ho, P.H., Xie, L.L., Shen, X.: Optimal Relay Station Placement in IEEE 802.16j Networks. In: IWCMC, Hawaii, USA (August 2007)

3. Lin, B., Ho, P.H., Xie, L.L., Shen, X.: Relay Station Placement in IEEE 802.16j Dual-Relay MMR Networks. In: IEEE ICC, Beijing, China (May 2008)

4. Cover, T.M., Gamal, A.A.E.: Capacity Theorems For The Relay Channel. IEEE Trans. on Inf. Theory 25(5), 572-584 (1979)

5. So, A., Liang, B.: Optimal Placement And Channel Assignment Of Relay Stations In Heterogeneous Wireless Mesh Networks By Modified Bender'S Decomposition. The Elsevier Ad Hoc Networks journal (invited from IFIP Networking 2007) 7(1), 118-135 (2009) 
6. So, A., Liang, B.: Enhancing WLAN Capacity by Strategic Placement of Tetherless Relay Points. IEEE Trans. on Mobile Computing 6(5), 522-535 (2007) (Extended version of paper in IFIP Networking 2006)

7. Amaldi, E., Capone, A., Cesana, M., Malucelli, F.: Optimization Models for the Radio Planning of Wireless Mesh Networks. In: Akyildiz, I.F., Sivakumar, R., Ekici, E., de Oliveira, J.C., McNair, J. (eds.) NETWORKING 2007. LNCS, vol. 4479, pp. 287-298. Springer, Heidelberg (2007)

8. Amaldi, E., Capone, A., Cesana, M., Filippini, I., Malucelli, F.: Optimization Models and Methods for Planning Wireless Mesh Networks. Computer Networks 52(11), 2159-2171 (2008)

9. Xie, L.L., Kumar, P.R.: A Network Information Theory for Wireless Communication: Scaling Laws and Optimal Operation. IEEE Trans. on Inf. Theory 50, 748-767 (2004)

10. Mark, J.W., Zhuang, W.: Wireless Communications and Networking. Prentice Hall, Englewood Cliffs (2003)

11. Garey, M.R., Johnson, D.S.: Computers and Intractability: A Guide to the Theory of NP-completeness, pp. 245-248. W. H. Freeman and Company, New York (1979)

12. IEEE Standard for Local and Metropolitan Area Networks Part 16: Air Interface for Fixed Broadband Wireless Access Systems, IEEE Std. 802.16-2004 (2004) 